\title{
Terapia com Laser de baixa potência para hipersensibilidade dentinária: eficácia de um protocolo
}

Low power Laser therapy for dentin hypersensitivity: efficacy of a protocol

Terapia con Láser de baja potencia para hipersensibilidad dentinaria: eficacia de un protocolo Wellington Raimundo da COSTA JÚNIOR ${ }^{1}$ Yallis Maria BARBOSA ${ }^{1}$

Vanda Sanderana Macêdo CARNEIRO²

Patrícia Lins Azevedo do NASCIMENTO ${ }^{3}$

${ }^{1}$ Curso de Graduação em Odontologia - Centro Universitário Tabosa de Almeida - ASCES UNITA - 55016-901 Caruaru-PE, Brasil

${ }^{2}$ Professora Adjunta da Faculdade de Odontologia de Pernambuco, Universidade de Pernambuco (FOP-UPE) Recife - PE, Brasil

${ }^{3}$ Professor Assistente III do Curso de Odontologia no Centro Universitário Tabosa de Almeida - ASCES UNITA - 55016-901 Caruaru-PE, Brasil

\section{Resumo}

A hipersensibilidade dentinária (HD)é uma condição clínica comum e dolorosa que promove um desconforto ao paciente. A HD é causada devido à exposição da dentina no meio bucal pela perda de esmalte e cementodecorrente de vários fatores. A Laserterapia de Baixa Potência (LBP) tem sido proposta como terapia para a HD.Este trabalho investigou a ação da irradiação com LBPno tratamento da HD. Foi realizado um ensaio clínico randomizado cego do tipo "Split mouth" em 21 pacientes, submetidos a dois tratamentos distintos: verniz fluoretado e laserterapia $\left(880 \mathrm{~nm} ; 100 \mathrm{~mW} ; 1 \mathrm{~J} / \mathrm{cm}^{2} ; 4\right.$ pontos; 1 sessão semanal por 4 semanas). O grau de sensibilidade foi avaliado por meio de Escala Visual Analógica (EVA) para o teste tátil com sonda exploratória e para a aplicação de ar comprimido. O LBP promoveu um efeito fotobiomodulador, apresentando uma remissão de $81,58 \%$ para dor provocada pelo estímulo tátil, sendo equivalente a ação promovida pelo verniz fluoretado (80\%). Sobre a dor provocada com o ar comprimido, houve uma remissão de dor de $50 \%$ no grupo submetido ao LBP, enquanto no grupo submetido ao verniz se obteve55,71\%. Conclui-se que o protocolo adotado para a LBP foi eficaz na remissão da dor provocada pela HD.

Descritores: Odontologia; Sensibilidade da Dentina; Materiais Dentários; Terapia com Luz de Baixa Intensidade.

\section{Abstract}

Dentin hypersensitivity (DH) is a common and painful clinical condition that promotes patient discomfort. DH is caused due to the exposure of the dentin in the oral medium by the loss of enamel and cement due to several factors. The Low Level Lasertherapy (LLLT) has been proposed as therapy for $\mathrm{DH}$. This work investigated the action of LLLT irradiation in the treatment of $\mathrm{DH}$. A randomized "Split mouth" clinical trial was performed in 21 patients, submitted to two different treatments: fluoride varnish and lasertherapy $(880 \mathrm{~nm} ; 100 \mathrm{~mW} ; 1 \mathrm{~J} / \mathrm{cm} 2 ; 4$ points; 1 weekly session for 4 weeks). The degree of sensitivity was evaluated by an Analog Visual Scale (AVS) both for the tactile test applied by an exploratory probe and for the application of compressed air. The LLLT promoted a photobiomodulation effect, presenting a remission of $81.58 \%$ for the pain caused by tactile stimulation, that was equivalent to the action promoted by the fluoride varnish (80\%). About the pain caused by the compressed air, there was a pain remission of $50 \%$ to the group submitted to LLLT, while the group submitted to the varnish obtained $55.71 \%$. It is concluded that the protocol adopted for LLLT was effective for the remission of pain caused by DH.

Descriptors: Dentistry; Dentin Sensitivity; Dental Materials; Low-Intensity Light Therapy.

\section{Resumen}

La hipersensibilidad dentinaria (HD) se trata de una condición clínica común y dolorosa que promueve una incomodidad al paciente. Comúnmente, es causada debido a la exposición de la dentina en el medio bucal por la pérdida de esmalte y cemento que puede ocurrir debido a varios factores. La Laserterapia de Baja Potencia (LBP) ha sido propuesta como terapia para la HD. Este trabajo se propuso investigar la acción de la irradiación con LBP (880nm, 100mW, 1J / cm2, 4 puntos / aplicación, 1 sesión semanal por 4 semanas) en el tratamiento de la HD. Se realizó un ensayo clínico aleatorizado ciego del tipo "Split mouth" en 21 pacientes, sometidos a dos tipos de tratamientos: barniz fluorado y láserterapia. El grado de sensibilidad fue evaluado por medio de Escala Visual Analógica (EVA) para la prueba táctil con sonda exploratoria y para la aplicación de aire comprimido. EI LBP promovió un efecto fotobiomodulador, con remisión del 81,58\% para el dolor provocado por el estímulo táctil, siendo equivalente a aquella por el barniz fluorado (80\%). En relación al dolor provocado con el aire comprimido, hubo una remisión de dolor del $50 \%$ en el grupo sometido al LBP, mientras que en aquel sometido al barniz fluorado fue del $55,71 \%$. Se concluye que la LBP fue eficaz en la remisión del dolor provocado por la HD.

Descriptores: Odontología; Sensibilidad de la Dentina; Materiales Dentales; Terapia Ligera de Baja Intensidad.

INTRODUÇÃO

A hipersensibilidade dentinária (HD) trata-se de uma condição dolorosa que promove um desconforto ao paciente. Esta é uma ocorrência clínica que está entre os problemas mais delicados e persistentes na Odontologia, sendo uma das mais comuns queixas com crescimento da prevalência entre os adultos ${ }^{1}$

Comumente, a HD é devida à exposição da dentina no meio bucal, correspondendo à perda de esmalte e do cemento. Essa perda da estrutura dental pode ocorrer devido a vários fatores: lesões cariosas, abrasão, erosão, recessão gengival, entre outros, até mesmo pela união de dois ou mais fatores ${ }^{2}$.

Atualmente a condição é explicada pela teoria da hidrodinâmica proposta por
Brannstromem 1964², teoria a qual, fala do deslocamento dos líquidos presentes nos túbulos dentinários causando a sensação dolorosa. A morfologia da dentina em pacientes sensíveis é modificada, os túbulos dentinários apresentam-se em quantidade maior e o seu diâmetro é aumentado ${ }^{3}$

Boa parte das abordagens terapêuticas atuais são para realização da vedação desses túbulos ou até mesmo a diminuição do fluxo de fluidos nos mesmos. São algumas delas: ajuste oclusal (diminuição da pressão intrapulpar, fazendo com que ocorra a obliteração dos túbulos dentinários); aplicação de adesivos dentinários e restaurações (esses materiais evitam o movimento do líquido presente nos túbulos pela sua obliteração; cirurgias 
mucogengivais (enxertos e retalhos periodontais recobrem as áreas radiculares com abfração e erosão) ${ }^{4}$. O sucesso da terapia para a HD depende do correto diagnóstico de sua causa, sendo assim, terá compreensão do comportamento desse desconforto que pode variar em cada indivíduo dependendo do dente afetado ${ }^{5}$.

O agente dessensibilizante tópico mais utilizado na odontologia é o flúor, que diminui a sensibilidade através do fechamento dos túbulos dentinários, tendo como mecanismo de ação a cristalização e redução dos líquidos para a polpa, como também a redução do diâmetro dos túbulos devido a sua união com os íons cálcio, formando fluoretos de cálcio. Para ser considerado ideal para o tratamento da HD, o agente dessensibilizante deve seguir o maior número de requisitos possíveis: possuir fácil aplicação, não ser irritante a polpa, agir rapidamente, possuir longo tempo de efetividade, não manchar os dentes e não causar dor ${ }^{5,6}$.

Outra forma de tratamento da hipersensibilidade e que está crescendo bastante é o uso do laser odontológico. LASER é um acrônimo da língua inglesa: Light Amplification by Stimulated Emission of Radiation e trata-se de uma radiação eletromagnética com características peculiares: comprimento de onda único, propagação coerentemente de ondas no espaço e no tempo, que carregam de forma colimada e direcional altas concentrações de energia. Inclusive, a radiação do laser interage com a matéria viva através dos processos ópticos de reflexão, transmissão, espalhamento e absorção ${ }^{7}$.

A laserterapiade baixa potência (LBP) tem sido proposta para tratamento da hipersensibilidade dentinária em consultório por conta de seus efeitos terapêuticos. A irradiação por laseres promove a fotobiomodulação da atividade celular, fazendo com que as células odontoblásticas aumentem sua síntese na deposição de dentina terciária, formem uma grande barreira contra estímulos térmicos, e assim evitem a transmissão de estímulos dolorosos pela polpa. Apesar dos vários estudos, seu mecanismo de ação não é completamente esclarecido ${ }^{8,9}$.

Além disso, como outros efeitos bioestimulantes do laser no processo de reparo tecidual destacam-se: incentivo à produção de colágeno, inibição da secreção de alguns mediadores químicos, modificação da densidade capilar e a estimulação da microcirculação local. Ressalta-se que o LBP não possui efeito diretamente curativo, mas atua como agente antiálgico, proporcionando ao organismo uma melhor resposta à inflamação, reduzindo o edema e minimizando a sintomatologia dolorosa, além de favorecer eficazmente a reparação tecidual da região lesada ${ }^{10}$.

Diante deste levantamento, este trabalho buscou averiguar a ação da irradiação com o laser de baixa potência no tratamento da hipersensibilidade dentinária para elaboração de protocolo de atendimento dessa condição nas Clínicas Odontológicas da ASCES- UNITA.

MATERIAL E MÉTODO

- Seleção dos casos clínicos

O estudo consistiu num ensaio clínico randomizado simples cego do tipo "Split mouth" com amostra de 21 pacientes dos quais 76 dentes foram submetidos à laserterapia de baixa potência (LBP) (G1) e 70 dentes submetidos ao tratamento controle com verniz fluoretado (G2) atendidos na clínica escola de Odontologia numa Instituição de Ensino Superior do interior de Pernambuco. O projeto foi submetido ao Comitê de Ética em Pesquisa Local e aprovado para a execução com o no do CAAE 60163016.2.0000.5203.O termo de Consentimento Livre e Esclarecido (TCLE) foi anexado as fichas clínicas dos pacientes envolvidos.

No quadrante bucal correspondente ao grupo experimental (G1), 76 dentes foram submetidos à laserterapia de baixa potência (LBP). Já nos quadrantes contralaterais, 70 dentes foram submetidos ao tratamento controle(G2) com Verniz de Flúor Fluorniz (SS White, Rio de Janeiro, RJ).Estes dentes foram avaliados quanto a sensibilidade dentinária através de dois testes: o teste tátil, no qual o estímulo mecânico foi realizado com sonda exploratória aplicada sobre a superfície dentinária da porção cervical coronária com leve pressão; e ainda um teste evaporativo, realizado através da aplicação de jato de ar da seringa tríplice a uma distância média de $10 \mathrm{~cm}$ durante 5 segundos. Dois operadores previamente calibrados realizaram os testes clínicos.

Para coleta de dados, utilizou-se como instrumento uma ficha clínica voltada para a avaliação da dor aos estímulos supracitados através da Escala Visual Analógica (EVA), que quantificava como 10 (dez) o pior quadro de dor e como 0 (zero) a ausência de dor. Os pacientes foram avaliados em cada sessão previamente a terapia designada realizada em 4 sessões com intervalos semanais.

Os critérios de exclusão deste estudo foram: pacientes já submetidos a tratamentos 
dessensibilizantes em consultório ou caseiros, restaurações em resina composta ou selamento dos túbulos com sistemas adesivos e/ou dentes anteriores.

\section{- Equipamento, material e procedimento}

O equipamento para LBP utilizado foi o Therapy XT (DMC do Brasil, São Carlos -SP) para irradiação de pré-molares e molares, conforme parâmetros estabelecidos: $\lambda=808 \mathrm{~nm}$; $\mathrm{P}=100 \mathrm{~mW} ; 4$ pontos de irradiação perpendicular ao longo eixo do dente (mesial, médio, distal e apical), $1 \mathrm{~J}$ /ponto, 4 aplicações com intervalos de 1 semana) e avaliação de resposta ao estímulo tátil e ao ar comprimido por meio da EVA.

Os elementos irradiados foram comparados com dentes contralaterais do mesmo paciente submetido a dessensibilização por Verniz de Flúor Fluorniz (SS White, Rio de Janeiro, RJ, Brasil). Ambos os tratamentos eram previamente avaliados e então aplicados e reavaliados semanalmente, durante 4 semanas. $\mathrm{Na}$ quinta semana, o paciente retornava apenas para reavaliação.

RESULTADOS

Conforme a obtenção do diagnóstico clínico da condição pesquisada; $90 \%$ dos participantes sentiam dor ao ingerir líquidos gelados e no ato mecânico da escovação. Somente $10 \%$ dos participantes da pesquisa sentiam dor apenas durante a escovação. 85,7 $\%$ dos pacientes relataram ainda fazer uso de escovas com cerdas duras e força durante a escovação.

Foram avaliados e tratados 21 pacientes sendo 16 do gênero feminino com idades entre 23 à 55 anos e 05 do gênero masculino com idade entre 20 à 43 anos de idade, dos quais 76 dentes foram submetidos à laserterapia de baixa potência (LBP) (G1) e 70 dentes submetidos ao tratamento controle com verniz fluoretado (G2).

$\mathrm{Na}$ primeira sessão previamente aos tratamentos, os dentes do grupo experimental tinham uma média de sensibilidade ao estímulo tátil com sonda exploradora de 2,61 pontos pela E.V.A.; enquanto a resposta ao ar comprimido foi quantificada com média de 5,32 pontos. Uma semana após a primeira irradiação com o LBP, a média das respostas ao estímulo tátil e ao ar comprimido foram 1,75 e 4,50 pontos da escala, respectivamente, havendo uma redução de 0,86 e 0,82pontos.

No grupo experimental, após um mês de avaliações semanais, $81,58 \%$ dos dentes apresentaram remissão da dor provocada ao estímulo tátil, tendo uma redução média de 1,97 pontos para esta variável.Com relação ao estímulo de dor provocada com 0 ar comprimido, os dentes apresentaram uma redução média de dor quantificada em 3,67 pontos pela E.V.A e uma remissão de dor equivalente à $50 \%$ conforme observado na Figura 1.

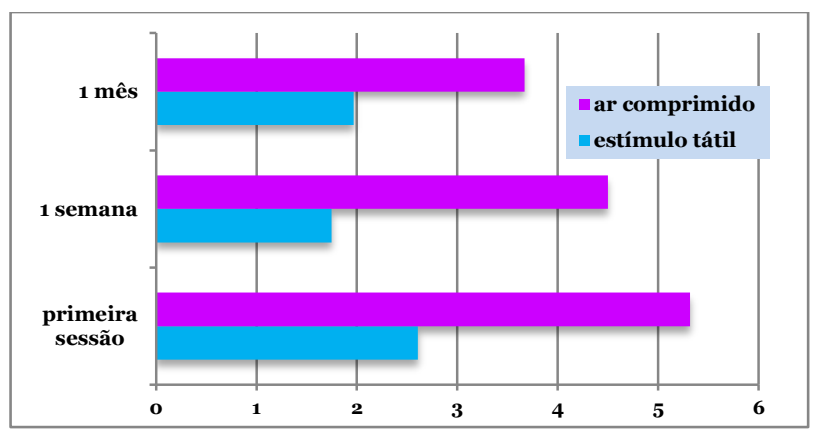

Figura 1: Média de sensibilidade ao estímulo tátil e ao ar comprimido na sessão prévia ao tratamento e uma semana após o mesmo e redução média de pontos de dor segundo a escala visual analógica após um mês de avaliações semanais.

Já no grupo controle, na primeira sessão que antecedeu os tratamentos, os dentes do grupo controle tinham uma média de sensibilidade ao estímulo tátil com sonda exploradora de 2,34 pontos pela E.V.A., enquanto a resposta ao estímulo ao ar comprimido foi quantificada com média 4,81 pontos. Uma semana após a primeira terapia controle com o uso do verniz fluoretado, a média das respostas aos estímulos supracitados foram de 1,74 e 3,48 respectivamente, havendo uma redução média de 0,6 para o primeiro estímulo e 1,33 para o segundo estímulo provocado.

Após um mês de acompanhamento, com sessões de avaliações semanais, houve grande redução da sensibilidade. Para $80 \%$ dos elementos dentários houve remissão completa de dor para o estímulo tátil, e os resultados obtidos foram redução de 1,87 pontos para o estímulo tátil e 3,62 para o estímulo ao ar comprimido segundo a E.V.A.; com uma remissão da dor de aproximadamente $55,71 \%$ para o último teste.

DISCUSSÃO

A hipersensibilidade dentinária é definida como uma dor aguda, de curta duração, localizada, que advém da dentina exposta a partir de abrasão, erosão, abfração ou desnudamento da raiz como resultado de uma recessão gengival ou tratamento periodontal ${ }^{11-15}$. A maioria (90\%) dos pacientes atendidos nessa pesquisa relatavam sentir dor ao ingerir líquidos gelados e no ato mecânico da escovação. O quadro dos pacientes estava relacionado à recessão gengival ocasionada por fatores como abrasão, abfração e uso de 
aparelho dentário, com grande parte dos pacientes $(85,7 \%)$ relatando o uso de escovas com cerdas duras e força durante a escovação. É extremamente importante que o cirurgiãodentista tenha conhecimento dos fatores etiológicos da hipersensibilidade dentinária, para que possa fornecer instruções básicas de higiene ao seu paciente, como a forma correta de realização de escovação para minimizar danos ao paciente ${ }^{16}$.

A dor pode ser definida como uma experiência sensorial e emocional desagradável, associada a uma lesão real ou potencial. Quando há a estimulação nervosa, um impulso é propagado e interpretado como dor quando chega ao cérebro. Por ser subjetiva, cada paciente possui um limiar de dor diferente, ou seja, é o momento em que o estímulo passa a ser percebido como doloroso e cada indivíduo responde de forma diferente ao mesmo. Como também, o limiar de tolerância é diferente, já que se refere ao ponto de tal intensidade que não pode ser mais suportada ${ }^{17}$. Embora haja inúmeros estudos na literatura, o mecanismo de dor transmitido da dentina para a polpa ainda não é totalmente compreendido. Entretanto, a teoria da hidrodinâmica, apesar de antiga, é a mais aceita pela comunidade científica, uma vez que se fundamenta na movimentação de fluidos no interior dos túbulos dentinários. Esse fluido consequentemente estimula fibras nervosas que transmitirão estímulos ao cérebro e este, por sua vez, responde na forma de dor ${ }^{5,16,18}$.

Por se tratar da dor um dado subjetivo da semiologia, uma vez que se trata de um sintoma relatado pelo avaliado, e que tem a interferência inclusive de componentes psicológicos e sociais na sua construção para um indivíduo, tornou-se necessário adotar uma ferramenta no sentido de quantificar este dado. Para isso, adotamos a escala visual analógica (EVA), ferramenta já estabelecida na literatura para este tipo de avaliação. Neste estudo observou-se que cada paciente possuía um limiar de dor diferente, e que mesmo dentes de uma mesma hemiarcada apresentavam escores discrepantes quando avaliados pela EVA, ou submetidos ao teste térmico e de ar comprimido durante as avaliações. A profilaxia prévia com pedra-pomes foi empregada nas sessões e esta etapa também promoveu o estímulo doloroso em vários voluntários. Porém, era necessária para remoção do biofilme dental impregnado na superfície a ser submetida aos distintos tratamentos. A maior intensidade de dor ocorreu sempre no início do tratamento, havendo remissão parcial relatada já na segunda e terceira sessão de avaliação do quadro.

Tanto no grupo controle quanto no experimental, foi perceptível que a dor ao estímulo ao ar comprimido foi mais intensa no tratamento do que o estímulo tátil. Isso é justificado pelo fato que o estímulo ao ar comprimido vai mais além do que a sensibilidade ao toque; já que, quando o mesmo é lançado sobre a dentina, exerce uma pressão sobre os túbulos dentinários e movimenta com maior vigor o fluido dessa área que reflete como dor, pois há ligeiros deslocamentos físicos de odontoblastos e nervos adjacentes. Essa estimulação mecânica libera $\mathrm{K}^{+}$no interior da célula dos odontoblastos despolarizando a membrana dos nervos e gerando impulsos nervosos ${ }^{19}$.

O mecanismo de ação do verniz fluoretado pode ser explicado de forma simples, a diminuição da sensibilidade é feita através do fechamento dos túbulos dentinários, ocorrendo após a aplicação do produto na superfície do dente, o verniz irá cristalizar e por consequência terá uma redução dos líquidos para a polpa e redução do diâmetro dos túbulos devido a sua união com os íons cálcio, formando fluoretos de cálcio $^{4,21,22}$. Nessa perspectiva, no presente estudo, já era esperado que o grupo controle obtivesse sucesso uma vez que o verniz fluoretado é considerado padrão ouro no tratamento da hipersensibilidade dentinária com eficácia comprovada cientificamente $e^{20-22}$.

Ainda, para que haja um efeito positivo do verniz fluoretado, é necessário que o paciente siga as instruções corretamente: se faz necessário que o paciente não faça a ingestão de alimentos e bebidas imediatamente após a sua aplicação, sendo liberado o consumo após 30 minutos. Além disso, o fabricante preconiza aplicação tópica em um período mínimo de um mês. A escovação é outro fator que pode interferir na eficácia do produto, foi recomendado aos pacientes que fizessem a escovação do local da aplicação do verniz somente após 24 horas. Segundo VALE E BRAMANTE $(1997)^{20}$, o produto faz adesão à superfície do elemento dentário, sendo facilmente removido com a escovação e com a própria saliva. A bula do verniz fluoretado utilizado nesse experimento recomenda que, no tratamento de dessensibilização da dentina, a aplicação deve ser feita por 2 ou 3 vezes a cada 7 dias, demonstrando o efeito em curta duração do produto.

O Laser é uma forma de energia que se transforma em energia luminosa visível ou não, e que vem ganhando espaço no mercado odontológico. O laser de baixa intensidade, em 
especial, embora não possua efeito diretamente curativo, proporciona analgesia, atuação antiinflamatória e ainda acelera o reparo tecidual ${ }^{6,10}$ Estudos como o de Gelskey et al. (1993) 24 Dantas et al.(2013) ${ }^{4}$, Aranha et al.(2003) ${ }^{26}$, Gerschman et al.(1994) ${ }^{25}$, embora tenham sido utilizados metodologias diferentes, apresentam em comum a efetividade da laserterapia; em ambos os estudos, houve uma diminuição da hipersensibilidade dentinária. $\mathrm{O}$ mecanismo de atuação desta terapia está baseado na indução de alterações na rede de transmissão nervosa dentro da polpa, produzindo desta forma dentina secundária, obliterando os canalículos dentinários. Este mecanismo impede que haja comunicação com a polpa e consequentemente estímulos dolorosos ${ }^{4,24-26}$ É importante ressaltar que está terapia pode ainda ser aplicada com outras, como a terapia por verniz fluoretado, sem que haja prejuízo no mecanismo de ação de ambas.

No presente estudo, a laserterapia apresentou resultados semelhantes ao do verniz fluoretado, que já é considerada uma terapia consagrada para o quadro de hipersensibilidade dentinária. Uma outra vertente que poderia justificar este resultado seria a subjetividade da dor, que pode apresentar relatos e quantificações distintos de um mesmo paciente quando em condições diferentes. A EVA é uma ferramenta ainda com limitações nesse sentido, pois não deixa de apresentar um componente subjetivo para quantificar o dado dor.

Há ainda um outro fator a ser avaliado para estes resultados, posto que além dos efeitos locais, o LBP apresenta ainda efeitos sistêmicos para o paciente irradiado, o que pode ter consistido num viés metodológico da pesquisa, posto que dentes que não foram irradiados também teriam 0 beneficiamento sistemático pela interferência nos mediadores da dor, como fator de necrose tumoral, ou mesmo o aumento da produção de energia através de um maior aporte de ATP para os tecidos ${ }^{10}$ Além disso, mostrou-se como uma terapia eficiente por não ser irritante à polpa, não acarretar dor ao paciente, ser de fácil aplicação, ser efetiva por um longo período e apresentar resultados semelhantes aos do verniz fluoretado, atual terapia mais aplicada no tratamento da hipersensibilidade dentinária.

A ação do laser é então mais abrangente do que a do verniz, uma vez que, quando utilizado nos parâmetros corretos, promove a formação de dentina esclerótica, obliterando os túbulos dentinários e diminuindo a transmissão neural. É importante que o Cirurgião-Dentista tenha o conhecimento desta terapia e do seu mecanismo de ação, além dos possíveis benefícios promovidos ao paciente para que aquele possa então diagnosticar e oferecer uma nova opção terapêutica para a hipersensibilidade dentinária cada dia mais prevalente nos consultórios odontológicos para que este quadro possa receber tratamento adequado e promover melhoria na qualidade de vida do paciente.

CONCLUSÃO

Conclui-se que o protocolo de LBP adotado foi eficaz na remissão do quadro de dor provocado pelos estímulos testados durante esse estudo. Houve uma maior remissão da dor ao estímulo tátil na EVA após a primeira aplicação, evidenciando que tratamento com laserterapia pode se mostrar mais eficaz que o verniz fluoretado num primeiro momento. Quanto ao estímulo provocado com 0 ar comprimido, os resultados do grupo controle foram superiores ao experimental.

\section{REFERÊNCIAS}

1. Lopes AO, Eduardo CP, Aranha ACC. Clinical evaluation of low-power laser and a desensitizing agent on dentin hypersensitivity. Lasers Med Sci. 2015;30:823-29.

2. Tonetto MR, Dantas AAR, Bortolini GF, Fabris $M$, Campos EA, Andrade M F. Hipersensibilidade dentinária cervical: Em busca de um tratamento eficaz. Rev Odontol Univ Cid São Paulo. 2012;24(3):190-99.

3. Brännström M. Sensory mechanism in dentine. Ed. In: Anderson JD. A hydrodynamic mechanism in the transmission of pain producing stimuli through the dentine. Oxford: Pergammon Press; 1963.

4. Querido MTA, Raslan SA, Scherma AP. Hipersensibilidade dentinária: revisão de literatura. R Period. 2010;20(2):39-46.

5. Peixoto LM, Daleprane B, Batitucci MHG, S anglard L, Pazinatto FB. Tratamento da Hipersensibilidade dentinária cervical. Rev Bras Pesq Saúde. 2010;12(2):69-74.

6. Dantas EM, Dantas PMC, Nobrega FJO, Vasconcelos RG, Aguiar Junior N, Queiroz LMG. Tratamento da hipersensibilidade dentinária cervical com laser de baixa potência. Odontol Clín.-Cien. 2013;12(1):7-11.

7. Cavalcanti $T M$, Almeida-Barros $R Q$, Catão MHCV, Feitosa APA, Lins RDAU. Conhecimento das propriedades físicas e da interação do laser com os tecidos biológicos na odontologia. An Bras Dermato. 2011;86(5): 955-60.

8. Moosavi H, Maleknejad F, Sharifi M, Ahrari F. A randomized clinical trial of the effect of low-level laser therapy before composite placement on 
postoperative sensitivity in class $\mathrm{V}$ restorations. Lasers Med Sci. 2015;30:1245-49.

9. Sgolastra F, Petrucci A, Severino M, Gatto R, Monaco A. Lasers for the Treatment of Dentin Hypersensitivity: A Meta- analysis. J Dent Res. 2013;92(6):492-99.

10.Lins RDAU, Dantas EM, Lucena KCR, Catão MHCV, Granville-Garcia AF, Carvalho Neto LG. Efeitos bioestimulantes do laser de baixa potência no processo do reparo. An Bras Dermatol. 2010;85(6):849-55.

11.Panagakos F, Schiff T, Guignon A. Dentin hypersensitivity: effective treatment with an inoffice desensitizing paste containing 8\% arginine and calcium carbonate. Am J Dent. 2009;25(3):165-70.

12. Amarasena N, Spencer J, Ou Y, Brennan D. Dentine hypersensitivity: australian dentists' perspective. Aust Dent J. 2010;55(2):181-87.

13. Sales ALLS. Avaliação in vitro da eficácia e durabilidade da ação de agentes dessensibilizantes na redução da permeabilidade dentinária [dissertação]. São José dos Campos: Universidade Estadual Paulista- UNESP; 2013.

14. Marín C, Bottan ER, Degobi BL, Magnani N. Efetividade de duas pastas dessensibilizantes no controle da hipersensibilidade dentinária em pacientes com recessão gengival. Odonto. 2013; 21(41-42):47-54.

15. Bernardo LP. Influência da raspagem e alisamento radicular na hipersensibilidade dentinária. Braz J Periodontol. 2013;23(1):32-8.

16. Oliveira JM, Oliveira M, Santos APMS, Vadillo JG, Campos CN, Chaves MGAM. Hipersensibilidade dentinária: considerações para o sucesso em seu manejo clínico. HU Rev. 2012;38(1-2):13-20.

17. Klaumann PR, Wouk AFPF, Sillas T. Patofisiologia da dor. Arch Veter Sci. 2008;13(1):1-12.

18. Hashim TN, Gasmalla BG, Sabahelkheir AH, Awooda AM. Effect of the clinical application of the diode laser $(810 \mathrm{~nm})$ in the treatment of dentine hypersensitivity. BMC Research Note. 2010;85(6):849-55.

19. Malamed, S.F Manual de anestesia local. 5. ed. Rio de Janeiro, Elsevier; 2005.

20.Vale IS, Bramante AS. Hipersensibilidade dentinária: diagnóstico e tratamento. Rev Odontol Univ Cidade de São Paulo. 1997;11(3):207-13.

21. Carvalho DM, Salazar M, Oliveira BH, Coutinho ESF. O uso de vernizes fluoretados e a redução da incidência de cárie dentária em préescolares: uma revisão sistemática. Rev Bras Epidemiol. 2010;13(1):139-49.

22. Oliveira DWD, Paiva SM, Cota LOM. Etiologia, epidemiologia e tratamento da hipersensibilidade dentinária: uma revisão de literatura. Braz J Periodontol. 2017;27(4): 76-85.

23. Cavalcante MS, Pereira TB, Tenório Neto JF, Santos NB, Ribeiro CMB, Batista, LHC. Redução da dor decorrente da hipersensibilidade dentinária cervical após dois tratamentos. Rev Dor. 2015;16(4):259-62.

24. Gelskey SC, White JM, Pruthi VK. The effectiveness of the $\mathrm{Nd}$ : YAG laser in the treatment of dental hypersensitivity. J Can Dent Assoc. 1993;59(4):377-86.

25. Gerschman JA, Ruben J, Gebart-Eaglemont J. Low-level laser therapy for dentinal tooth hypersensitivity. Aust Dent J. 1994;39(6): 353-57.

26. Aranha ACC, Pimenta LAF, Marchi GM. Avaliação clínica de métodos de dessensibilização dentinária em lesões cervicais. ABLO News. 2003;4:19.

27. Silva ETC, Vasconcelos RG, Vasconcelos MG. Uso e eficácia clínica do laser no tratamento da hipersensibilidade dentinária: uma revisão de literatura. Arch Health Invest. 2019;8(10): 638-43.

\section{CONFLITO DE INTERESSES}

Os autores declaram não haver conflitos de interesse

AUTOR PARA CORRESPONDÊNCIA

Patrícia Lins Azevedo do Nascimento

Av. Portugal, 584, Bairro Universitário

55016-901, Caruaru-PE, Brasil

E-mail: patricianascimento@asces.edu.br 